\title{
Closure of patent foramen ovale for secondary prevention of cryptogenic stroke: current perspectives
}

Rakhee R. Makhija ${ }^{1}$, Chandrasekar Palaniswamy ${ }^{2}$, Wilbert S. Aronow ${ }^{3}$

${ }^{1}$ Department of Medicine, Regional Medical Center, San Jose, CA, USA ${ }^{2}$ Department of Medicine, Division of Cardiology, UCSF Fresno Medical Education Program, Fresno, CA, USA

${ }^{3}$ Department of Medicine, Division of Cardiology, New York Medical College at Westchester Medical Center, Valhalla, NY, USA

Submitted:17 March 2018

Accepted: 25 June 2018

Arch Med Sci 2020; 16 (5): 1243-1246

DOI: https://doi.org/10.5114/aoms.2019.81744

Copyright $\odot 2019$ Termedia \& Banach

Patent foramen ovale (PFO) is found in about $25 \%$ of the population from autopsy studies. In patients with cryptogenic stroke (which accounts for about $30 \%$ of strokes), the prevalence of PFO is even higher (about 40-50\%). In patients younger than 55 years of age with cryptogenic stroke, the odds ratio for the prevalence PFO is $6(95 \% \mathrm{Cl}: 3.72-9.68)$, suggesting a very strong association between the two [1]. Over the past decade, PFO closure has been studied as a non-pharmacologic means of secondary prevention of stroke. The earlier devices used for PFO closure were the CardioSEAL and STARFlex devices (NMT Medical, Boston, MA). Currently, the Amplatzer PFO Occluder (Abbott, St. Paul, MN) and Gore Cardioform septal occluder (W. L. Gore \& Associates, Inc, Flagstaff, AZ) are widely used. Essentially, these devices have a double-disc design with left and right atrial discs, deployed percutaneously through a femoral venous approach.

Earlier trials [2-4] had failed to show a significant benefit of PFO closure over medical therapy. Accordingly, in 2014, the American Heart Association/American Stroke Association guidelines recommended against routine use of PFO closure for secondary prevention of cryptogenic stroke in patients with a PFO without evidence of deep venous thrombosis [5]. However, recent trials [6-9] have shown superiority of PFO closure over medical therapy alone to prevent recurrent strokes in this population. The essential details of the individual trials are summarized in Table I. Recently published meta-analyses have also confirmed the findings from the recent trials. In a meta-analysis of 2892 patients enrolled in 4 randomized control trials [3, 6-8], PFO closure decreased the absolute risk for recurrent stroke by $3.2 \%$ (risk difference: $-0.032 ; 95 \% \mathrm{Cl}:-0.050$ to $-0.014)$ compared with medical therapy [10]. In another meta-analysis that included 3440 patients enrolled in 5 major randomized trials [2, $3,6-8]$, PFO closure significantly reduced recurrent stroke $(O R=0.41$; $95 \% \mathrm{Cl}: 0.19-0.90 ; p=0.03)$ compared to medical therapy alone [11]. Of note, new onset atrial fibrillation was significantly more frequent after PFO closure (OR $=5.75,95 \% \mathrm{Cl}: 3.09-10.70 ; p<0.00001)$.

What should the current evidence-based practice in patients with PFO and cryptogenic stroke be? Interpretation of conflicting results from individual trials is compounded by significant heterogeneity in study population, medical therapy and the type of device used for closure. However, this heterogeneity also provides us the opportunity to find out the

\author{
Corresponding author: \\ Prof. Wilbert S. Aronow MD, \\ FACC, FAHA \\ Cardiology Division \\ Westchester Medical \\ Center and \\ New York \\ Medical College \\ Macy Pavilion \\ Room 141 \\ Valhalla, NY 10595, USA \\ Phone: (914) 493-5311 \\ Fax: (914) 235-6274 \\ E-mail:wsaronow@aol.com
}




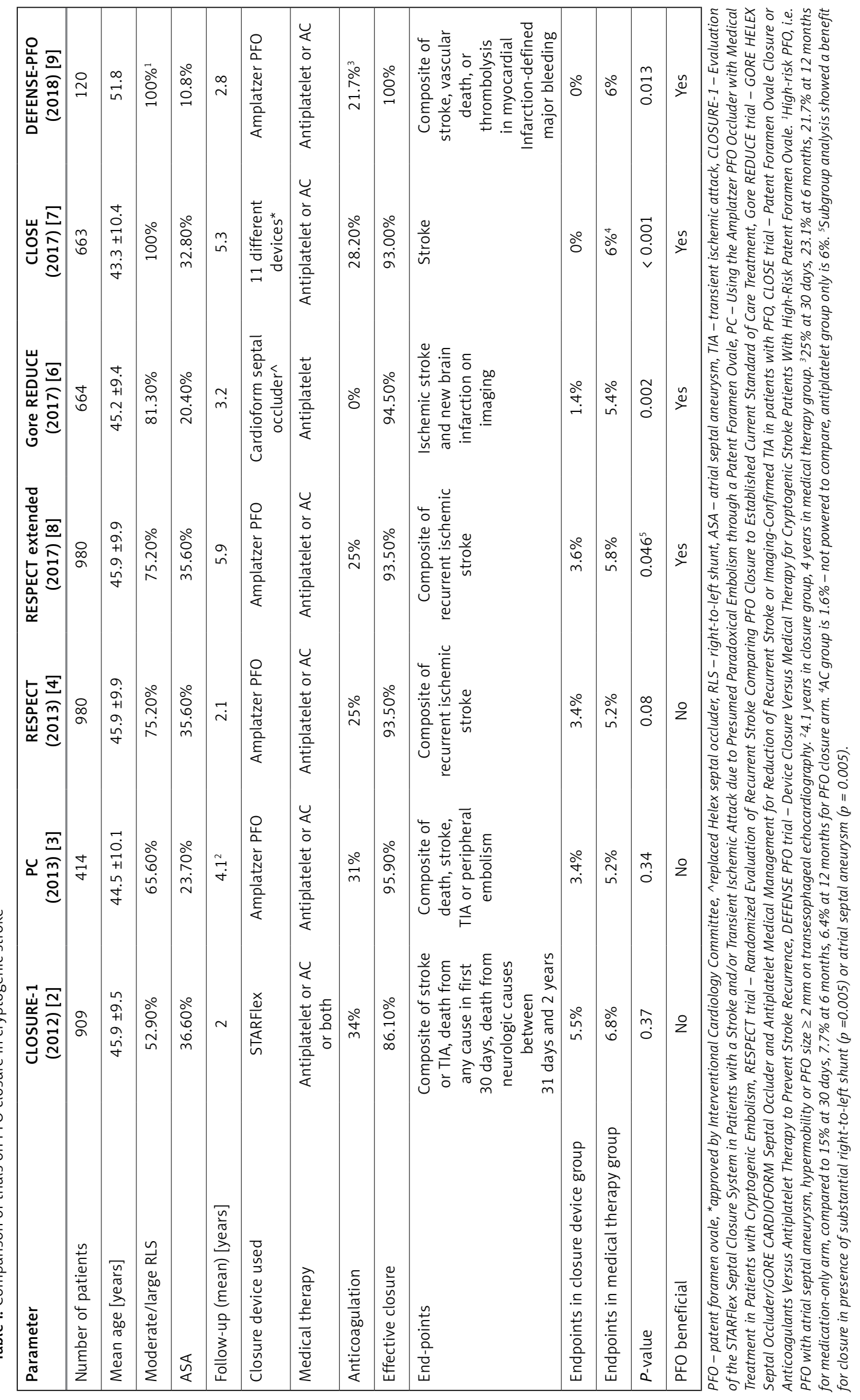


subset of the population for which PFO closure is potentially effective.

First, let us discuss the differences in patient selection. The benefit of PFO closure is noted when patients with more significant shunting were included. For instance, about $80 \%$ of patients in the Gore REDUCE trial [6] (Gore Helex Septal Occluder/GORE CARDIOFORM Septal Occluder and Antiplatelet Medical Management for Reduction of Recurrent Stroke or Imaging-Confirmed TIA in Patients With PFO) and over $75 \%$ of patients in the RESPECT trial [4, 8] (Randomized Evaluation of Recurrent Stroke Comparing PFO Closure to Established Current Standard of Care Treatment) had at least a moderate degree of right-to-left shunt through the PFO. Similarly, in the CLOSE trial [7] (Patent Foramen Ovale Closure or Anticoagulants Versus Antiplatelet Therapy to Prevent Stroke Recurrence), all patients had at least a moderate right-to-left shunt. In the recently published DEFENSE-PFO trial (Device Closure Versus Medical Therapy for Cryptogenic Stroke Patients With High-Risk Patent Foramen Ovale) [9], all patients had high-risk PFO, i.e. size $\geq 2 \mathrm{~mm}$, PFO with atrial septal aneurysm, or hypermobility (phasic septal excursion into either atrium $\geq 10 \mathrm{~mm}$ ) on transesophageal echocardiography.

Patent foramen ovale can be classified as small (<2 mm), medium (2-3.9 mm), or large ( $\geq 4 \mathrm{~mm})$. On bubble study, the shunt can be classified as small ( $\leq 5$ bubbles), moderate (6-25 bubbles), or severe ( $>25$ bubbles). Patients with a large PFO or with a moderate-to-severe shunt have an increased risk of recurrent stroke and are more likely to benefit from device closure [12]. Atrial septal aneurysm, as defined by excursion of the interatrial septum by $10 \mathrm{~mm}$ or greater, is independently associated with an increased risk of recurrent stroke. This can sometimes be associated with a large PFO and lower rates of successful closure. Patients with lacunar stroke, caused by small vessel disease of the brain, are not likely to benefit from PFO closure. Of the earlier trials, only the RESPECT trial [4] had excluded patients presenting with lacunar infarction. The PC trial (Using the Amplatzer PFO Occluder with Medical Treatment in Patients with Cryptogenic Embolism) and CLOSURE I trial [2] (Evaluation of the STARFlex Septal Closure System in Patients with a Stroke and/or Transient Ischemic Attack due to Presumed Paradoxical Embolism through a Patent Foramen Ovale) had also included patients with TIA which were not necessarily cryptogenic in nature.

Second, we will discuss the differences in medical therapy in the control arm. In the earlier trials, medical therapy consisted of antiplatelets and/or anticoagulants, which was based on the discretion of the treating physician. In the new- er trials, only antiplatelet agents were exclusively used in the control arm, which is consistent with current established stroke guidelines. Although in the CLOSE trial [7] there was a separate arm of patients with anticoagulant use, there was lower than expected patient enrollment, and this group was underpowered to make any meaningful comparisons. In the DEFENSE-PFO trial [9], the decision on anticoagulation was based on the treating physician's discretion (for example, at 6 months, $7.7 \%$ of patients in the PFO closure arm versus $23.1 \%$ in the control arm were on warfarin).

Third, there were significant differences in the type of device used. The STARFlex device that was originally used in the CLOSURE-1 trial is already off the market due to inferior efficacy (effective closure was $86.1 \%$ ) and concerns with safety. The Amplatzer PFO Occluder and Gore Cardioform septal occluder that have superior efficacy were used in the recent trials.

Fourth, the earlier trials were underpowered to detect any significant benefit with PFO closure. The PC trial suffered from recruitment and dropout issues. The follow-up period in CLOSURE-1 and RESPECT trials was only about 2 years (as compared to 5.9 years in the RESPECT extended trial and 5.3 years in the CLOSE trial).

The Risk of Paradoxical Embolism (RoPE) Score [13] was originally developed in 2011 to identify patients with cryptogenic stroke and a PFO in whom PFO was likely to be the cause of their stroke. Components of the score include age (0-5 points, with younger age assigned more points), cortical infarct on imaging, absence of smoking, hypertension, diabetes mellitus, and prior stroke/ TIA (all the above assigned 1 point each). The higher the score, the more likely the stroke is related to paradoxical embolism from the PFO. Although this has not been validated or studied in PFO closure, it is prudent to use this score to screen out patients with minimal potential benefit (especially a score of 0-3 where the stroke is likely from other etiologies). One of the main drawbacks with the score is that the size of the PFO or severity of shunt is not factored into this calculation.

Our suggested approach is as follows: PFO closure should not be routinely performed in all patients with cryptogenic stroke. In younger patients (less than 55 years of age) with cryptogenic stroke and a high risk PFO (moderate to severe right-toleft shunt; atrial septal aneurysm; increased atrial septal excursion $\geq 10 \mathrm{~mm}$; PFO size $\geq 2 \mathrm{~mm}$ ), PFO closure significantly reduces the risk of recurrent stroke compared to medical therapy alone. Of note, a higher incidence of atrial fibrillation was seen in the PFO closure group in most trials. However, when overall serious adverse events were compared, there was no significant difference be- 
tween the two groups. Candidacy for anticoagulation if atrial fibrillation were to occur after device implantation should be a consideration when patients are being evaluated for this procedure.

In conclusion, we suggest shared decision-making with the patient, explaining the likelihood that the stroke is caused by the PFO, and discussing the evidence regarding efficacy and risks of the procedure.

\section{Conflict of interest}

The authors declare no conflict of interest.

\section{References}

1. Overell JR, Boe I, Lees KR. Interatrial septal abnormalities and stroke: a meta-analysis of case-control studies. Neurology 2000; 55: 1172-9.

2. Furlan AJ, Reisman M, Massaro J, et al. Closure or medical therapy for cryptogenic stroke with patent foramen ovale. N Engl J Med 2012; 366: 991-9.

3. Meier B, Kalesan B, Mattle HP, et al. Percutaneous closure of patent foramen ovale in cryptogenic embolism. N Engl J Med 2013; 368: 1083-91.

4. Carroll JD, Saver JL, Thaler DE, et al. Closure of patent foramen ovale versus medical therapy after cryptogenic stroke. N Engl J Med 2013; 368: 1092-100.

5. Kernan WN, Ovbiagele B, Black HR, et al. American Heart Association Stroke Council, Council on Cardiovascular and Stroke Nursing, Council on Clinical Cardiology, and Council on Peripheral Vascular Disease. Guidelines for the prevention of stroke in patients with stroke and transient ischemic attack: a guideline for healthcare professionals from the American Heart Association/American Stroke Association. Stroke 2014; 45: 2160-236.

6. Søndergaard L, Kasner SE, Rhodes JF, et al. Gore REDUCE Clinical Study Investigators. Patent Foramen Ovale Closure or Antiplatelet Therapy for Cryptogenic Stroke. N Engl J Med 2017; 377: 1033-42.

7. Mas JL, Derumeaux G, Guillon B, et al. CLOSE Investigators. Patent foramen ovale closure or anticoagulation vs. antiplatelets after stroke. N Engl J Med 2017; 377: 1011-21.

8. Saver JL, Carroll JD, Thaler DE, et al.; RESPECT Investigators. Long-term outcomes of patent foramen ovale closure or medical therapy after stroke. N Engl J Med 2017; 377: 1022-32.

9. Lee PH, Song JK, Kim JS, et al. Cryptogenic stroke and high-risk patent foramen ovale: the DEFENSE-PFO trial. J Am Coll Cardiol 2018; 71: 2335-42.

10. Shah R, Nayyar M, Jovin IS, et al. Device closure versus medical therapy alone for patent foramen ovale in patients with cryptogenic stroke: a systematic review and meta-analysis. Ann Intern Med 2018; 168: 335-42.

11. Schulze V, Lin Y, Karathanos A, et al. Patent foramen ovale closure or medical therapy for cryptogenic ischemic stroke: an updated meta-analysis of randomized controlled trials. Clin Res Cardiol 2018; 107: 745-55.

12. Lee JY, Song JK, Song JM, et al. Association between anatomic features of atrial septal abnormalities obtained by omni-plane transesophageal echocardiography and stroke recurrence in cryptogenic stroke patients with patent foramen ovale. Am J Cardiol 2010; 106: 129-34.
13. Wessler BS, Kent DM, Thaler DE, et al. The RoPE score and right-to-leftshunt severity by transcranial Doppler in the CODICIA study. Cerebrovasc Dis 2015; 40: 52-8. 